\title{
Patient sexual function and hip replacement surgery: A survey of surgeon attitudes
}

\author{
Rita Th. E. Harmsen ${ }^{1,2,3}$ • Melianthe P. J. Nicolai ${ }^{2}$ • Brenda L. Den Oudsten ${ }^{4}$. \\ Hein Putter ${ }^{5}$ - Tsjitske M. Haanstra ${ }^{1}$ - Peter A. Nolte ${ }^{6}$ Barend J. Van Royen ${ }^{1}$. \\ Henk Elzevier ${ }^{2}$
}

Received: 7 October 2016/Accepted: 23 March 2017 / Published online: 27 April 2017

(C) The Author(s) 2017. This article is an open access publication

\begin{abstract}
Purpose To explore practises of orthopaedic surgeons (and residents) in addressing sexual function (SF) in patients before and after total hip arthroplasty (THA).

Methods A 26-item questionnaire was sent to health professionals $(n=849) ; 526(62.0 \%)$ responses were included in the analyses.

Results About $78 \%$ of the respondents (77.5\%) almost never addressed SF. The most mentioned reason was that "patients do not ask" (47.4\%) followed by "I am not aware of possible needs" (38.6\%). SF was even less discussed (25.9\%) in elderly patients ( $>60$ years). The beneficial effect of THA on SF was rated the highest in retired surgeons $(p \leq 0.001)$, in which male surgeons scored higher than female surgeons
\end{abstract}

The work was performed at the Department of Urology and Orthopaedics, University Medical Center Leiden, The Netherlands.

\section{Rita Th. E. Harmsen}

Orthofit1@icloud.com; r.t.e.harmsen@lumc.nl

Melianthe P. J. Nicolai

melianthenicolai@gmail.com

Brenda L. Den Oudsten

B.L.denOudsten@uvt.nl

Hein Putter

h.putter@lumc.nl

Tsjitske M. Haanstra

t.haanstra@vumc.nl

Peter A. Nolte

peteranolte01@me.com

Barend J. Van Royen

BJ.vanRoyen@vumc.nl $(p=0.002)$. The importance of sexual dificulties (SD) in the decision to undergo surgery was rated lowest by residents $(p=0.020)$. Rating the risk for dislocation varied between occupations $(p=0.008)$ and gender $(p=0.016)$, female surgeons rated highest (median 5); $54.1 \%$ indicated the orthopaedic surgeon is responsible for providing information about the safe resumption of sexual activity.

Conclusions Surgeons show little attention to SF related issues in THA patients, which seems not in accordance to patients' needs. Addressing SF increases throughout a surgeon's career. There were divergent views and there is no "common advice" about the safe resumption of sexual activity. The results emphasize the need for guidelines and training in order to encourage addressing SF both, before and after THA.
Henk Elzevier

H.W.Elzevier@lumc.nl

1 Department of Orthopaedic Surgery, VU University Medical Center, De Boelelaan 1117, 1081 BT Amsterdam, The Netherlands

2 Department of Urology, University Medical Center, Albinusdreef 2, 2333 ZA Leiden, Netherlands

3 Department of Orthopaedic Surgery, Albinusdreef 2, 2333ZA Leiden, The Netherlands

4 Department of Medical and Clinical Psychology, University Center of Research on Psychological and Somatic Disorders (CoRPS), Warandelaan 2, 5037 AB Tilburg, The Netherlands

5 Department of Medical Statistics, University Medical Center, Albinusdreef 2, 2333 ZA Leiden, The Netherlands

6 Department of Orthopaedic Surgery, Spaarne Gasthuis, Spaarnepoort 1, 2134 TM Hoofddorp, The Netherlands 
Keywords Arthroplasty $\cdot$ Replacement hip · Osteoarthritis hip · Communication $\cdot$ Physician-patient relations $\cdot$ Sexual (Dys)function

\section{Introduction}

Each year more than one million patients worldwide undergo total hip arthroplasty (THA) for symptomatic hip arthritis (HA) [1]. Lavernia et al. (2015) found that HA interfered with sexual function (SF) in $82 \%$ of THA patients (mean age 65 ; range 20-89). Authors suggest SF should be routinely addressed with all patients undergoing THA [2]. Within fifty years of research, only a few studies have examined the impact of HA on SF and improvement of SF after THA [3-9]. Since 1991, Stern et al. (1991) found that nearly $80 \%$ of patients (who were satisfied with the THA result) felt the need for more information about SF afterwards; and in $20 \%$ sexual dysfunction (SD) had been an argument to undergo THA [4].

To our knowledge, there are only two studies published addressing SF in THA patients [7, 10]. However, these studies are small with less attention for specific views on patients' perspectives and safety matters.

In this context, the objectives of this study were to: (i) to explore practises of orthopaedic surgeons in addressing issues of sexual function (SF) in patients before and after total hip athroplasty (THA), (ii) surgeons' views on patients' perspectives of SF related issues, and (iii) surgeons' opinions on safe return to sexual activity after THA. Differences between the surgeons' gender and occupations (residents, practising surgeons, and retired surgeons) are of interest, in order to provide useful information to encourage communication about SF in future daily orthopaedic practice.

\section{Methods}

We conducted a cross-sectional survey among a group of orthopaedic surgeons with detailed measurements of SF related issues. We collected surgeons' opinions on patient perspectives, communication, and questions about safety matters, especially related to the safe resumption of sexual activity after THA and the surgical technique.

\section{Development of questionnaire}

A 28-item Dutch questionnaire was developed by an urologist (HE) for questioning medical disciplines; and previously used in cardiology, radiotherapy, oncology, nephrology [11-15].

This questionnaire was modified for use in orthopaedic practice by three authors (RH, $\mathrm{PN}, \mathrm{TH})$, and piloted on eight orthopaedic surgeons, five retired surgeons and 12 residents. Two questions were removed. It covers demographic questions (questions 1-7) and questions on the three objectives: (i) surgeons' views on patients' perspectives of SF related issues (questions 8-11); (ii) surgeons' practises in addressing SF issues and perceived barriers to communication (question 12-16); and (iii) surgeons' opinions on safe return to sexual activity after THA (question 17-22). Finally, there were some additional questions (questions 23-26). An in English translated version can be found in Appendix 1.

\section{Surgeons and procedure}

The 26-item modified questionnaire was posted to practising orthopaedic surgeons performing hip surgery $(n=455)$, retired orthopaedic surgeons $(n=149)$, and orthopaedic residents $(n=245)$ in the Netherlands. Addresses were retrieved from the member list of the Netherlands Orthopaedic Association (Nederlandse Orthopedische Vereniging, NOV). After screening on "performing hip surgery" and "living in the Netherlands" 849 addresses were retrieved. Two reminders were sent after six and 12 weeks. Data were collected and analysed anonymously. For research not involving patients, approval from an ethical board is not required in the Netherlands. Figure 1 shows the procedure for the selection of eligible respondents and response rates.

\section{Statistical analysis}

Statistical analyses were performed using IBM SPSS, version 22 for Mac/Windows. Most responses were scored on a visual analogue scale (VAS) ranging from no effect (1) to the strongest possible effect (10). For some questions an 'I do not know' option was available which was coded as ' 0 ' in the analyses.

The results are presented using descriptive analyses. Continuous variables were found to be not normally distributed and are therefore summarized as median (interquartile range IQR). Distributional differences between the occupations and genders were tested using Pearson's chi-squared tests or MannWhitney tests and Kruskal-Wallis test. Missing data were excluded from the percentage calculations; $p$-values of $<0.05$ were considered to be statistically significant.

\section{Results}

Of the 849 questionnaires sent out, $600(70.7 \%)$ were returned. Of these, 74 respondents chose not to participate in the study. Reasons for non-participation were: no longer actively performing surgery $(n=43 ; 58.1 \%)$, lack of experience $(n=21 ; 28.4 \%)$, not relevant $(n=6 ; 8.1 \%)$, not interested $(n=3 ; 4.1 \%)$, and no time $(n=1 ; 1.4 \%)$. In total 526 respondents were included in the analysis (62.0\%) Table 1. 


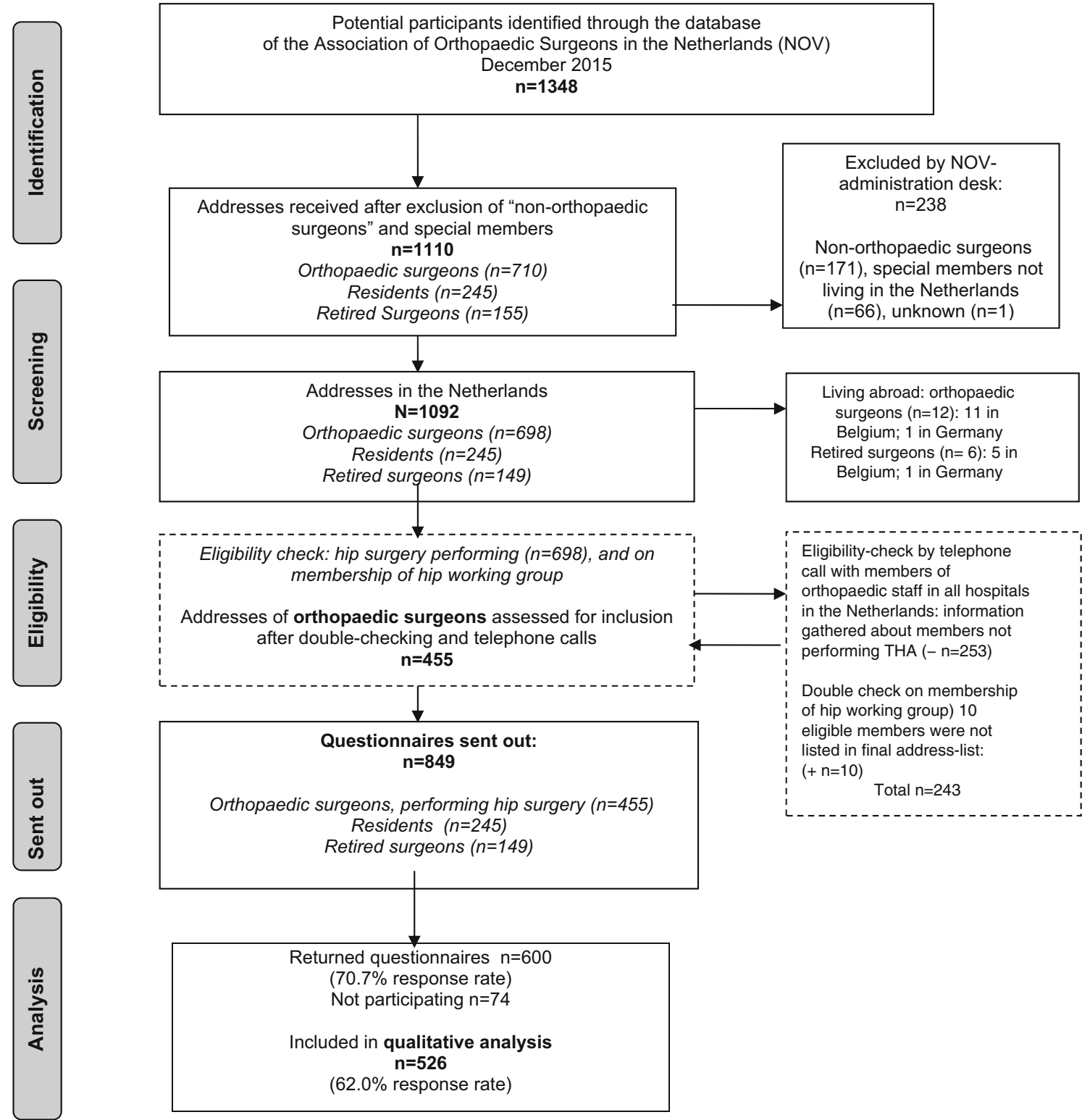

Fig. 1 Flowchart of the study procedure

\section{Views on patients' perspectives of SF related issues}

Table 2A shows the respondents' views regarding four questions: (i) the impact of $\mathrm{HA}$ on SF, (ii) improvement of SF after THA, (iii) the importance of SD in the decision to undergo surgery, and (iv) the need for information on the safe resumption of sexual activity. To each of those four questions, approximately $10 \%$ responded with " $d o$ not know" (range 7.0-13.5\%). The beneficial effect of THA on SF was rated the highest in retired surgeons $(p \leq 0.001)$, in which male surgeons scored higher than female surgeons $(p=0.002)$. The importance of SD in the decision to undergo surgery was rated lowest by residents $(p=0.020)$.

\section{Opinions on a safe return to sexual activity after THR}

Table 2B shows surgeons' opinions about six factors considered to be of influence in patients' safe resumption of intercourse. Approximately 3\% of the respondents did not answer to all questions (missing range: 5-34). Compared to all categories of orthopaedic surgeons, residents thought more often that "age" influences safe resumption $(p=0.001)$. For per-operative stability the distribution differed between the occupations $(p=0.001)$, although the medians were equal.

Rating the risk for dislocation within the first three months, 69 chose the option "I do not know" (13.1\%). The total cohort rated the risk at median 3 (IQR 2-6). The rating varied widely 
Table 1 Characteristics of the respondents

\begin{tabular}{|c|c|c|c|c|}
\hline Cohort-information & $\begin{array}{l}\text { "Overall" } \\
n=526(100 \%) \\
\mathrm{n}(\% \text { of total })\end{array}$ & $\begin{array}{l}\text { Practising surgeons } \\
n=326(52 \%) \\
\mathrm{n}(\% \text { of total })\end{array}$ & $\begin{array}{l}\text { Residents } \\
n=123(23.4 \%) \\
\mathrm{n}(\% \text { of total })\end{array}$ & $\begin{array}{l}\text { Retired surgeons } \\
n=77(14.6 \%) \\
\mathrm{n}(\% \text { of total })\end{array}$ \\
\hline \multicolumn{5}{|l|}{ Gender } \\
\hline Male surgeons & $467(88.8)$ & $300(92.0)$ & $90(73.2)$ & \multirow[t]{2}{*}{$77(100)$} \\
\hline Female surgeons & $59(11.2)$ & $26(8.0)$ & $33(26.8)$ & \\
\hline \multicolumn{5}{|l|}{ Age-groups } \\
\hline 20-30 year & $32(6.1)$ & $1(0.3)$ & $31(25.2)$ & - \\
\hline $31-40$ year & $192(36.5)$ & $100(30.7)$ & $92(74.8)$ & - \\
\hline $41-50$ year & $97(18.4)$ & $97(29.8)$ & - & - \\
\hline $51-60$ year & 89 (16.9) & $88(27.0)$ & - & $1(1.3)$ \\
\hline $61-70$ year & $79(15.0)$ & $36(11.0)$ & - & $43(55.8)$ \\
\hline$>70$ year & $37(7.0)$ & $4(1.2)$ & - & $33(42.9)$ \\
\hline \multicolumn{5}{|l|}{ Type of hospital/clinic } \\
\hline University & $60(11.4)$ & $16(4.9)$ & $36(29.3)$ & $8(10.4)$ \\
\hline General teaching & $224(42.6)$ & $121(37.1)$ & $70(56.9)$ & $33(42.9)$ \\
\hline Regional/district & $193(36.7)$ & $154(47.2)$ & $5(4.1)$ & $34(44.2)$ \\
\hline Specialized/private & $43(8.2)$ & $33(10.1)$ & $8(6.5)$ & $2(2.6)$ \\
\hline$\geq 2$ clinics & $6(1.1)$ & $2(0.6)$ & $4(3.3)$ & - \\
\hline \multicolumn{5}{|l|}{ Experience } \\
\hline $0-11$ months & $25(4.8)$ & $4(1.2)$ & $21(17.1)$ & - \\
\hline $1-2$ year & $61(11.6)$ & $10(3.1)$ & $51(41.5)$ & - \\
\hline $3-5$ year & $108(20.5)$ & $59(18.1)$ & $49(39.8)$ & - \\
\hline $6-10$ year & $70(13.3)$ & $70(21.5)$ & & \\
\hline $11-15$ year & $58(11.0)$ & $58(17.8)$ & - & - \\
\hline $15-25$ year & $78(14.8)$ & $66(20.2)$ & $2(1.6)$ & $10(12.8)$ \\
\hline$>25$ year & $125(23.8)$ & $58(17.8)$ & - & $66(87.0)$ \\
\hline "Retired" & $1(0.2)$ & & & $1(0.2)$ \\
\hline \multicolumn{5}{|c|}{ Number of THR performed per year } \\
\hline$<50$ & $198(37.6)$ & $70(21.5)$ & $114(92.7)$ & $14(18.2)$ \\
\hline $50-100$ & $214(40.7)$ & $172(52.8)$ & $9(7.3)$ & $33(42.9$ \\
\hline $100-200$ & $96(18.3)$ & $71(21.8)$ & - & $25(32.5)$ \\
\hline$>200$ & $18(3.4)$ & $13(4.0)$ & - & $5(6.5)$ \\
\hline \multicolumn{5}{|l|}{ Surgical technique } \\
\hline Posterior/postero-lateral & $313(59.5)$ & $204(62.6)$ & $69(56.1)$ & $40(51.9)$ \\
\hline Anterior/anterolateral & $63(12.0)$ & $42(12.9)$ & $11(8.9)$ & $10(13.0)$ \\
\hline Direct lateral & $104(19.8)$ & $62(19.0)$ & $22(17.9)$ & $20(26.0)$ \\
\hline Various $(\geq 2)$ & $46(8.7)$ & $18(5.2)$ & $21(17.1)$ & $7(9.1)$ \\
\hline
\end{tabular}

THA total hip arthroplasty

between occupations: median for practising surgeons: 3 (IQR 2-5); for residents: 4 (IQR 3-6); and for retired surgeons: 4 (IQR 2-6.50) $(p=0.008)$, and also across gender: males: 3 (IQR 2-5); females: 5 (IQR 3-6.50) $(p=0.016)$.

Overall, $7.4 \%(n=39)$ reported knowledge of patients who had experienced dislocation caused by sexual activity; a further $5.5 \%(n=29)$ suspected this. One third $(33.1 \% ; n=174)$ indicated that resuming was advisable whenever the patient felt ready. This was most often advised by surgeons who practised an anterior approach (48.4\%) compared to those who performed a posterior $(32.3 \%)$ or direct-lateral approach $(29.8 \%)(p=0.024)$. Recommendation to wait six to eight weeks after surgery was responded by $42.5 \%(n=223 / 525)(p=0.008)$. In case of per-operative instability of the implant, $19 \%$ would address precautions on safely resuming; $39.7 \%$ of respondents would do so only when patients would ask for. 


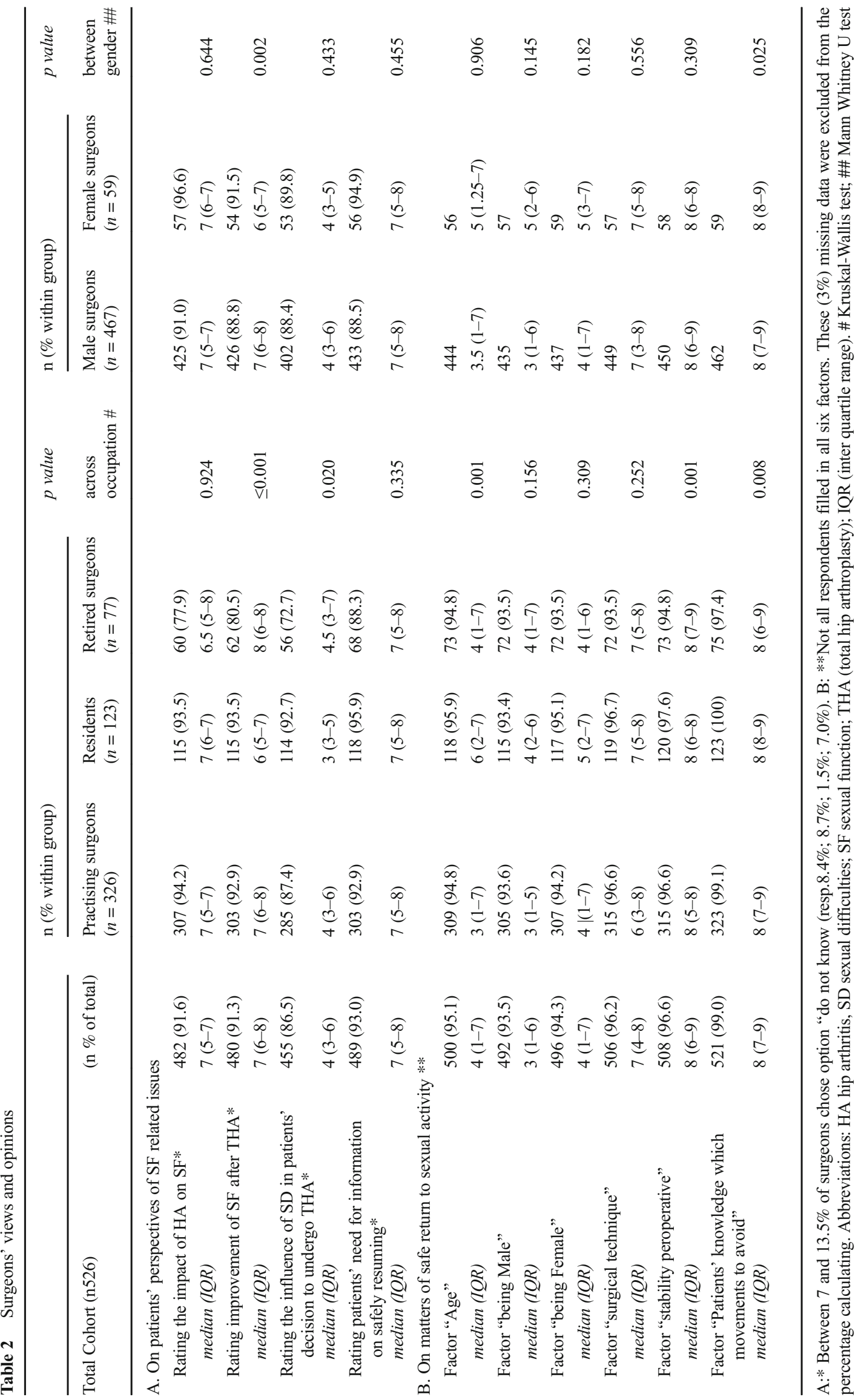




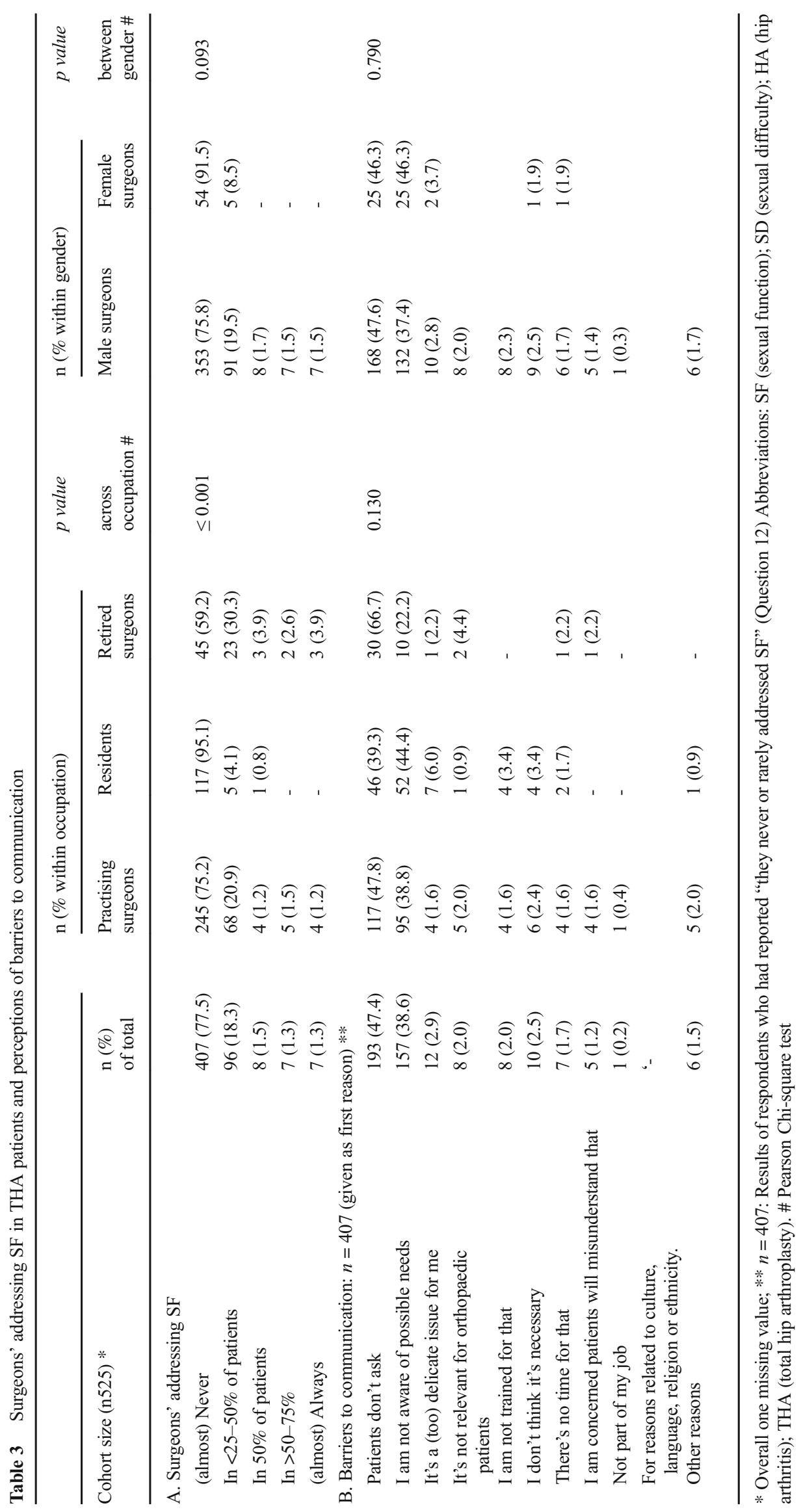




\section{Perceptions of barriers to communication}

Table 3 summarizes the responses towards communication. Retired surgeons had addressed SF more often $(41.8 \%)$ compared to residents $(4.9 \%)$ and practising surgeons $(24.8 \%)(p \leq 0.001)$. We asked respondents who rarely address $\mathrm{SF}$, to rank three out of eleven possible barriers. The most mentioned barrier was that "patients do not ask" (47.4\%) followed by "I am not aware of possible needs" $(38.6 \%)$.

Almost 90\% ( $n=467)$ of the respondents reported that in discussing SF, patients' gender is not relevant. Of the 56 respondents who thought that gender could be an issue, discussing SF with female patients was perceived as more difficult in $8.6 \%(45 / 523)$ than with male patients $(2.1 \%)$. Distribution on gender showed that in addressing SF, 9.5\% (44/464) of male surgeons perceived female patients as more difficult, whereas $8.5 \%(5 / 59)$ of female surgeons perceived male patients as more difficult.

Addressing SF with senior patients $>60$ years of age was considered to be difficult in $25.9 \%$ (135/522): residents scored highest $(44.3 \%$; 54/122) compared to practising surgeons $(23.8 \% ; 77 / 324)$ and retired surgeons $(5.3 \% ; 4 / 76)$ $(p \leq 0.001)$. Female surgeons $(37.3 \%)$ were less inclined to discuss SF with patients $>60$ years compared to male surgeons $(24.4 \%)(p=0.103)$.

A total of $284(54.1 \%)$ respondents indicated that the orthopaedic surgeon was primarily responsible for addressing SF with patients before and after THA. Residents more often suggested nurse practitioners were responsible $(19.5 \%)$ than did orthopaedic $(15.0 \%)$ and retired surgeons $(11.8 \%)(p=0.002)$. The need for additional training in addressing SF was reported by $52.1 \%$ of respondents $(p \leq 0.001)$. Twenty-six percent did not consider SD as a relevant issue for hip patients in their practice, and $32.1 \%$ did not know $(p=0.026)$. Over half of respondents $(55.1 \%)$ agreed that PROM questionnaires should include SF $(p=0.013)$.

\section{Discussion}

Surgeons show little attention to SF related issues in their THA patients. However, attention increases throughout career. We found divergent views and no "common advice" about safe resumption of sexual activity. Advices seem independent to surgical approach. Respondents rated the risk for dislocation during SA rather low.

\section{Limitations and strengths of the study}

The questionnaire was not psychometrically tested before use; this may have led to some shortcomings in validity and reliability, variables could have been misunderstood due to lack of formulating definitions. We suggest there were missing values for this reason in question 17 (3\%). Not all respondents filled in second and third reasons (question 13). We, therefore, chose to analyse the first reason, only. Secondly, the cohort studied, is probably not generalizable. Sex-related issues are sometimes a 'taboo' topic for some cultures, considering that this activity may be seen as forbidden or sacred based on religious beliefs or morals. Therefore, the results should be considered as best-case estimates, not applicable to other populations.

Nevertheless, overall, this study contains very few missing values. Despite the inevitable risk of response and information bias, this study offers a high response rate, especially for this type of (sensitive) investigation. Furthermore, it benefits from a broad overview among attitudes and views of orthopaedic surgeons to SF related issues in THA patients, per occupation as well as per gender.

Addressing SF was difficult for $77.5 \%$ of the respondents and this finding is in line with the two available, previous studies: in the UK 69.0\% [7] and in the USA $80.0 \%$ [10]. However, we found that retired surgeons had addressed SF more often (40.8\%) than residents (4.9\%), practising surgeons $(24.8 \%)$, and female surgeons were less inclined to address SF (91.5\%) as compared to their male colleagues $(75.8 \%)$. That was a somewhat unexpected finding in view of previous research: Birkhoff et al. (2016) found that female physicians address a taboo topic (as sexual abuse) more frequently than do their male colleagues [16], and Bertakis (2009) reports about a more devoted attitude in female physicians (internal and general) spending more time to psychosocial counselling compared to their male colleagues, who were more technically oriented [17]. Although communication about SF in orthopaedic literature is limited [18], the importance of effective communication skills in the patient-doctor relationship is widely recognized [19].

We looked for barriers in communication. Although the most cited reason was because patients are not initiating SF issues themselves; the patients' age ( $>60$ years) was of influence too $(25.9 \%)$. Interestingly, the factor no time was not indicated to be important (1.7\%) compared to approximately $40 \%$ of respondents in other area's of medical disciplines [11, $13,14,16]$. It has been noted that patients do not raise the subject spontaneously [20]. We suggest surgeons should find effective standardized ways to provide "easy" communication about $\mathrm{SF}$ in their practises.

In an earlier systematic review, we published about improvements of sexual activity after THA $(\Delta 0-77 \%)$; and the patients' need for more advice (range 57-89\%) [18]. For $20 \%$ of the patients, SF appears to be an argument to undergo THA $[4,6]$. It is important to know patients' needs, motives and expectations about SF, and before starting the surgical 
procedure. Especially, since literature suggests that unfulfilled expectations will lead to dissatisfaction [21]. Even more, several studies indicate that some patients $(2-17 \%)$ never resume sexual activity again after THA $[6,9,22,23]$. It seems to be highly important to have better insights into the determinants of SF in THA patients.

The patients' fear for dislocation has been emphasized (up to $80 \%$ ) in previous literature [8]. In addition, the female patients in this study changed their preferred sexual positions after THA in non-recommended positions, mostly due to difficulties with the leg position [8]. Unknown is if this would lead to more dislocations of the prosthesis more easily. We had expected to find an association between the preferred technique and the surgeons' advice concerning the waiting time before resuming intercourse, however, we did not. One third of the respondents indicated that resuming was permitted whenever the patient felt ready, and this was unrelated to the surgical technique. This seems in line with a recent review stating that "a more liberal lifestyle restrictions and precautions protocol will not lead to worse dislocation rates, but instead will lead to earlier and better resumption of activities and higher patient satisfaction" [24].

To the best of our knowledge there are no studies focused on dislocation caused by intercourse and positions. Compared to 20\% $(n=254)$ of the USA surgeons [10], in our study a surprisingly low proportion of respondents reported being aware of at least one patient experiencing THA dislocation during sexual activity (7.4\%). Only one study has determined -theoretically, based on MRI, 3D studies, and animations- which sexual positions pose the greatest risk for impingement and thus for dislocation of the prosthesis [25]. Notwithstanding this, we asked surgeons if they would inform the patient about the risk for dislocation during sexual activity in case they noted during surgery that the stability of the prosthesis was suboptimal. Previous literature suggests that, in the event of instability patients should be informed about which sexual positions to avoid [1]. However, more than two thirds of respondents stated they would not inform the patients, or only if patients were to ask about it. Obviously, the majority of respondents reported that they routinely provide their patients with general information on how to prevent dislocation; probably supposing their patients can translate this into knowledge about safe sexual positions themselves. Therefore, it remains uncertain if indirect information puts patients into risk. Although, in the twentieth century, communication about SF still is difficult (from the perspective of both surgeon and patient), surgeons should look for standardized ways to provide patientinformation and tailor-made advice both, before and after surgery. In line with this, we believe that evaluating SF by means of PROMs could help to encourage surgeons to address SF, and will shed light on this under-recognized issue in orthopaedic practice.

\section{Conclusions}

Despite research, which suggests patients want more information and discussion with their surgeons about SF and hip replacement surgery, the majority of Dutch orthopaedic surgeons surveyed appear to not address this need. Our research did however show that addressing SF increases throughout a surgeon's career. It was also clear that the age of both, the surgeon and patient influences this discussion. Surgeons' views were divergent and there was no "common advice" about safe resumption of sexual activity. The results emphasize the need for further research and guidance for surgeons and their team in order to encourage addressing SF both, before and after THA.

HA, hip (osteo)arthritis; IQRs, interquartile ranges; PROMs, patient-reported outcome measures; SD, sexual dysfunction; SF, sexual function; SQoL, sexual quality of life; THA, total hip arthroplasty; VAS, visual analogue scale.

Acknowledgements We gratefully acknowledge the general peercoaching role of Pieter Schillemans, orthopaedic surgeon at Knee Clinic Amsterdam, The Netherlands; Tom Hogervorst, orthopaedic surgeon at Haga Hospital, The Haque, The Netherlands, for his advice and coordinating role in the pilot period; Alison Edwards, $\mathrm{PhD}$ for her language editing; and Peter Wall, orthopaedic surgeon University of Warwick Coventry, UK, for reading the final manuscript.

Authors' contributions Authors' contributions are as follows: - RH, BvR, TH, PN en HE contributed to the conception/design of the study;

- RH collected the data and wrote the manuscript.

- Data analysis and interpretation of data was done by RH, HP, MN, PN.

- Statistically evaluation was done by RH, HP.

- Proofreading was performed by all authors MN, PN, BdO, TH, BvR, HE and HP;

- All authors critically revised the manuscript for important intellectual content.

The work was performed at the Department of Urology, University Medical Center Leiden.

\section{Compliance with ethical standards}

Conflict of interest The authors declare that they have no conflict of interest.

Funding There is no funding source.

Ethical approval This article does not contain any studies with human participants or animals performed by any of the authors.

Informed consent In the Netherlands for research not involving patients or interventions, approval by an ethical board is not required. The questionnaire did not compromise orthopaedic surgeons' integrity, nor could respondents be identified. For that reason an informed consent, from all individual participants included in the study was not requested. Participants received a letter explaining the purposes of the study and the guarantee of anonymity, and decided wheter to participate or not. 


\section{Appendix 1}

\begin{tabular}{|c|c|}
\hline & $\begin{array}{l}\text { ou do not wish to fill in this } \\
\text { cate why: }\end{array}$ \\
\hline ० & I don't perform hip surgery \\
\hline o & Not relevant \\
\hline o & No time \\
\hline ० & Not interested \\
\hline ० & Lack of experience \\
\hline ० & Overly sensitive issue \\
\hline o & Other reason, namely... \\
\hline
\end{tabular}

\section{PERSONAL INFORMATION}

1 . What is your gender?
$0 \quad$ Male
$0 \quad$ Female

2. What is your age?
$0 \quad 20-30$
$0 \quad 31-40$
$0 \quad 41-50$
$0 \quad 51-60$
$0 \quad 61-70$

3. What is your occupation?
0 Orthopaedic surgeon
0 Resident
0 Orthopaedic surgeon/educator
0 Retired orthopaedic surgeon

4. How many hip operations do you perform each year?
$0 \quad$ Fewer than 50
0 Between 50 and 100
$0 \quad$ Between 100 and 200
$0 \quad$ More than 200

5. What is your surgical approach?
0 Posterior
0 Anterior
0 Direct-lateral
$0 \quad$ Other, namely.

6. Where do you (mainly) perform surgery?
0 Academic hospital
0 General teaching hospital
0 Regional or district hospital
0 Specialized or private clinic

7. How long have you been performing total hip replacement (THA) surgery?
$0 \quad 0-11$ months
$0 \quad 1-2$ years
0 6-10 years
$0 \quad 3-5$ years
$0 \quad 11-15$ years
$0 \quad 15-25$ years
$0>25$ years 


\section{PATIENTS PERSPECTIVES}

8. How would you rate the impact of hip arthritis (HA) on patients' sexual function (SF)? $(1=$ not important; $10=$ extremely important $)$

\begin{tabular}{llllllllll}
1 & 2 & 3 & 4 & 5 & 6 & 7 & 8 & 9 & 10 \\
\hline
\end{tabular}

9. How would you rate the beneficial effect of total hip arthroplasty (THA) on SF?

( $1=$ none, $10=$ extremely high $)$

\begin{tabular}{llllllllll}
1 & 2 & 3 & 4 & 5 & 6 & 7 & 8 & 9 & 10 \\
\hline 0 & I don't know
\end{tabular}

10. How would you rate the importance of patients' desire to improve their sexual function (SF) in their decision to undergo surgery?

$(1=$ not important, $10=$ extremely important $)$

\begin{tabular}{llllllllll}
1 & 2 & 3 & 4 & 5 & 6 & 7 & 8 & 9 & 10 \\
\hline
\end{tabular}

0 I don't know

11. How would you rate the need for information on the safe resumption of sexual activity?

$(1=$ no need, $10=$ extremely high need $)$

\begin{tabular}{llllllllll}
1 & 2 & 3 & 4 & 5 & 6 & 7 & 8 & 9 & 10 \\
\hline
\end{tabular}

0 I don't know

\section{COMMUNICATION}

12. Do you discuss questions concerning SF in patients with HA and after THA?

0 Rarely/never

0 With fewer than $25-50 \%$ of patients

$0 \quad$ With around $50 \%$ of patients

0 With more than $50-75 \%$ of patients

0 (Almost) always

13. If you "rarely or never" discuss questions concerning SF with patients, what is the reason for this?

Please rate the most important three factors, with 1 as the most important.

$0 \quad$ I am not aware of possible needs

$0 \quad$ Patients don't ask

$0 \quad$ It's not part of my job

0 There's no time for it

$0 \quad$ It's a too delicate issue for me

0 I don't think it's necessary

$0 \quad$ I'm not trained for that

$0 \quad$ I'm concerned patients will misunderstand that

$0 \quad$ It's not relevant for orthopaedic patients

0 For reasons related to culture, language, religion or ethnicity

$0 \quad$ Other reason, namely..........

14. In discussing SF issues, does the patient's gender make a difference?

$0 \mathrm{No}$, gender makes no difference

0 Yes, I find it more difficult to discuss this with women

0 Yes, I find it more difficult to discuss this with men

15. In discussing SF issues, does the patient's age make a difference?

0 No, age makes no difference

0 Yes, I find it more difficult to discuss this with senior people (60+)

0 Yes, I find it more difficult to discuss this with younger people (under 60) 
16. In your opinion, who is responsible for answering patients' questions about resumption of SF after THA?

0 Orthopaedic surgeon

0 General practitioner

0 Nurse practitioner/physician assistant

0 Physiotherapist

0 Nobody

0 Other, namely

\section{SAFETY MATTERS}

17. Please indicate, on a scale from $1-10$, which of the factors below matter in relation to the safe resumption of sexual activity after THA? $(1=$ not important, $10=$ extremely important $)$
0
Age $\quad$\begin{tabular}{llllllllll}
1 & 2 & 3 & 4 & 5 & 6 & 7 & 8 & 9 & 10 \\
\hline
\end{tabular}
$0 \quad$ Male patient

\begin{tabular}{llllllllll}
1 & 2 & 3 & 4 & 5 & 6 & 7 & 8 & 9 & 10 \\
\hline
\end{tabular}
\begin{tabular}{llllllllllll}
0 & Female patient & 1 & 2 & 3 & 4 & 5 & 6 & 7 & 8 & 9 & 10 \\
\hline
\end{tabular}
\begin{tabular}{lllllllllll}
0 & Surgical approach 1 & 2 & 3 & 4 & 5 & 6 & 7 & 8 & 9 & 10 \\
\hline
\end{tabular}

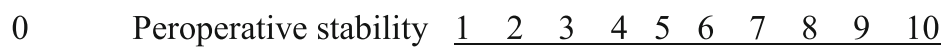
\begin{tabular}{llllllllllll}
0 & Knowledge of movements to avoid & 1 & 2 & 3 & 4 & 5 & 6 & 7 & 8 & 9 & 10 \\
\hline
\end{tabular}

18. How would you rate the risk of dislocation when resuming sexual activity within the first three months after the operation? $(1=$ no risk, $10=$ extremely high risk $)$

\begin{tabular}{llllllllll}
1 & 2 & 3 & 4 & 5 & 6 & 7 & 8 & 9 & 10 \\
\hline 0 & I don't know & & & & & &
\end{tabular}

19. Do you think information will matters in reducing risks?
$0 \quad$ Yes
$0 \quad$ No

20. If the hip is stable and the patient were to ask your advice on when sexuality can be safely resumed, what would your response be?

0 As soon as the patient is ready

0 After 2-4 weeks

0 After 6-8 weeks

0 After 3 months

0 After 6 months

21. If patients were to ask your advice on safe positions when resuming sexuality, what would your response be? (Please choose one answer that fits best.)

0 I'd refer them to the Internet

$0 \quad$ I'd refer them to a general practitioner

0 I'd refer them to a nurse practitioner/physician assistant

$0 \quad$ I'd refer them to a physiotherapist

0 I'd give them a flyer

0 I'd explain how to prevent dislocation

0 I'd tell them there are no restrictions whatsoever 
22. If you sense during the operation that stability is suboptimal, would you inform your patient about the risk of dislocation during sexual activity?

0 Yes

0 No

0 Only if patients ask

0 Other, namely

\section{GENERAL}

23. Have you ever come across a patient with a dislocation of the hip caused by sexual activity?
$0 \quad$ Yes
0 Probably, but I didn't ask
0 No

24. Do you think questionnaires used for Patient Reported Outcome Measures (PROMs) should include items on SF?
0 Yes
0 No
0 Don't know

25. Do you see sexual dysfunction (SD) as a relevant issue for hip patients in orthopaedic practice?
0 Yes
0 No
0 Don't know

26. If research were to demonstrate that $\mathrm{SD}$ is an important issue for hip patients, would you feel the need for training dedicated to this subject?
0 Yes
0 No

Open Access This article is distributed under the terms of the Creative Commons Attribution 4.0 International License (http:// creativecommons.org/licenses/by/4.0/), which permits unrestricted use, distribution, and reproduction in any medium, provided you give appropriate credit to the original author(s) and the source, provide a link to the Creative Commons license, and indicate if changes were made.

\section{References}

1. Meiri R, Rosenbaum TY, Kalichman L (2014) Sexual function before and after Total hip replacement: narrative review. Sex Med 2:159-167. doi:10.1002/sm2.35

2. Lavernia CJ, Villa JM (2015) High rates of interest in sex in patients with hip arthritis. Clin Orthop Relat Res 474:293-292. doi:10. 1007/s11999-015-4421-8

3. Todd RC, Lightowler CD, Harris J (1973) Low friction arthroplasty of the hip joint and sexual activity. Acta Orthop Scand 44:690-693

4. Stern SH, Fuchs MD, Ganz SB et al (1991) Sexual function after total hip arthroplasty. Clin Orthop Relat Res 269:228235

5. Meyer H, Stern R, Fusetti C et al (2003) Sexual quality-of-life after hip surgery - springer. J Orthop Traumatol 4:21-25

6. Laffosse J-M, Tricoire J-L, Chiron P, Puget J (2008) Sexual function before and after primary total hip arthroplasty. Jt Bone Spine 75:189-194. doi:10.1016/j.jbspin.2007.05.006
7. Wall PDH, Hossain M, Ganapathi M, Andrew JG (2011) Sexual activity and total hip arthroplasty: a survey of patients' and surgeons' perspectives. Hip Int 21:199-205

8. Yoon B-H, Lee K-H, Noh S et al (2013) Sexual activity after Total hip replacement in Korean patients: how they do, what they want, and how to improve. Clin Orthop Surg 5:269-277. doi:10.4055/ cios.2013.5.4.269

9. Nunley RM, Nam D, Bashyal RK et al (2015) The impact of Total joint arthroplasty on sexual function in young, active patients. J Arthroplast 30:335-340. doi:10.1016/j.arth.2014.09.029

10. Dahm DL, Jacofsky D, Lewallen DG (2004) Surgeons rarely discuss sexual activity with patients after THA; a survey of members of the American Association of hip and Knee Surgeons. Clin Orthop Relat Res 428:237-240. doi:10.1097/01.blo.0000137555. 74475.a5

11. Krouwel EM, Hagen JH, Nicolai MPJ et al (2015) Management of sexual side effects in the surgical oncology practice: a nationwide survey of Dutch surgical oncologists. Eur J Surg Oncol 41:11791187. doi:10.1016/j.ejso.2015.06.009

12. Krouwel EM, Nicolai MP, van der Wielen GJ et al (2015) Sexual concerns after (pelvic) radiotherapy: is there any role for the radiation oncologist? J Sex Med 12:1927-1939. doi:10.1111/jsm. 12969

13. van Ek GF, Krouwel EM, Nicolai MPJ et al (2015) Discussing sexual dysfunction with chronic kidney disease patients : practice patterns in the Office of the Nephrologist Discussing Sexual Dysfunction with chronic kidney disease. J Sex Med 12:2350 2363. doi:10.1111/jsm.13062 
14. Korse NS, Nicolai MPJ, Both S et al (2016) Discussing sexual health in spinal care. Eur Spine J 25:766-773. doi:10.1007/ s00586-015-3991-1

15. Nicolai MPJ, Both S, Liem SS et al (2013) Discussing sexual function in the cardiology practice. Clin Res Cardiol 102:329-336. doi: 10.1007/s00392-013-0549-2

16. Birkhoff EML, Krouwel EM, Nicolai MPJ et al (2016) Dealing with patients facing a history of sexual abuse: a cross-sectional survey among Dutch general practitioners. Eur J Gen Pract 22: 126-133. doi:10.3109/13814788.2015.1122752

17. Bertakis KD (2009) The influence of gender on the doctor-patient interaction. Patient Educ Couns 76:356-360. doi:10.1016/j.pec. 2009.07.022

18. Harmsen RTE, Haanstra TM, Sierevelt IN et al (2016) Does total hip replacement affect sexual quality of life? BMC Musculoskelet Disord 198:1-17. doi:10.1186/s12891-016-1048-1

19. Dibbelt S, Schaidhammer M, Fleischer C, Greitemann B (2009) Patient-doctor interaction in rehabilitation: the relationship between perceived interaction quality and long-term treatment results. Patient Educ Couns 76:328-335. doi:10.1016/j.pec.2009.07.031

20. Tavares Queiroz de Almeida PH, De Castro Ferreira C, Kurizky PS et al (2015) How the rheumatologist can guide the patient with rheumatoid arthritis on sexual function. Rev Bras Reumatol 55: 458-463 . doi:10.1016/j.rbr.2014.08.009 English Ed

21. Tilbury C, Haanstra TM, Leichtenberg CS et al (2015) Unfulfilled expectations after Total hip and knee arthroplasty surgery: there is a need for better preoperative patient information and education. $\mathrm{J}$ Arthroplast 31:2139-2145. doi:10.1016/j.arth.2016.02.061

22. Nordentoft T, Schou J, Carstensen J (2000) Changes in sexual behavior after orthopedic replacement of hip or knee in elderly males, a prospective study. Int J Impot Res 12:143-146

23. Klit J, Jacobsen S, Schmiegelow V et al (2015) Alternative outcome measures in young total hip arthroplasty patients : a prospective cohort study. HIP Int 25:152-159. doi:10.5301/ hipint. 5000200

24. van der Weegen W, Kornuijt A, Das D (2015) Do lifestyle restrictions and precautions prevent dislocation after total hip arthroplasty? A systematic review and meta-analysis of the literature. Clin Rehabil 30(4):329-339. doi:10.1177/0269215515579421

25. Charbonnier C, Chagué S, Ponzoni M et al (2014) Sexual activity after Total hip arthroplasty: a motion capture study. J Arthroplast 29:640-647. doi:10.1016/j.arth.2013.07.043 